\title{
Die Größe des öffentlichen Sektors - ein Benchmarking- Projekt: Methodologischer Erfahrungsbericht
}

\author{
Alfred Franz \\ Statistik Österreich
}

\begin{abstract}
Zusammenfassung: Der Staatssektor hat in modernen Volkswirtschaften außerordentliche Bedeutung erlangt, als Dienstleiter und Arbeitgeber, als Träger einer riesigen Umverteilungsmaschinerie, als Investor und Erhalter der Infrastrukturen, wie als Garant öffentlicher Ordnung und Sicherheit. Sich einen Überblick dieser machtvollen Stellung und der Erscheinungsvielfalt zu verschaffen ist immer auch ein quantitatives Problem, und umso mehr, wenn es um Vergleiche verschiedener Länder geht. Hierfür werden von der Wissenschaft Kennzahlen empfohlen, als ein Bündel von statistischen Indikatoren zur standardisierten Beschreibung der verschiedenen Aufgaben- und Organisationsformen: Staatsquoten i.w.S..

In dem hier vorgestellten Forschungsprojekt geht es um konzeptive u.a. statistische Erfordernisse des internationalen Vergleiches. Die Situation auf der Seite der statistischen Quellen ist allerdings nicht eben günstig. Trotz einer langen Entwicklung von internationaler Standardisierung, vor allem im Rahmen der Volkswirtschaftlichen Gesamtrechnung, sind die unmittelbaren Ergebnisse (,rohe“ Staatsquoten) jedenfalls adjustierungsbedürftig (,systematische Bereinigungen“).

Die praktische Bearbeitung hat rasch gezeigt, dass die erforderlichen Bereinigungen einen erheblichen Umfang annehmen und im Ergebnis systematisch zu beachten sind, auch wenn die Vorschläge beim status quo der Quellenlage nur exemplarischen Charakter haben können.
\end{abstract}

Abstract: In contemporaneous economies the government sector is outstanding for its role as provider of services and employer, or as central agent of a vast redistribution machinery or as investor in the infrastructure, and as ever, as the focus of public order and safety. Any attempt to get an overview of this extraordinary and varied function becomes quickly a quantitative problem, and particularly so if international comparison of this kind is at issue. Science has provided a kit of concepts on key numbers to statistically describe the usual variety of tasks and organisations in a more or less standardized way (government ratios, in a wider sense).

Starting from theoretical concepts but attempting empirical evaluation also. In the research project here presented, international comparison of this kind is the main focus. Unfortunately the situation in statistical practice is less favourable than suggested by theory. In spite of long-lasting endeavours of that kind (in particular from the National Account's side), the outcome of any such quota almost invariably needs substantial adjustment.

Therefore the evaluations have quickly shown that the adjustments needed are of significant size and must be consistently taken into account. 
However, the present proposals are no more than of an exemplary nature, because of the still prevailing insufficiencies of the given statistical sources.

Schlüsselwörter: Öffentlicher Sektor/Staatssektor, Messprobleme, Staatsquoten, Internationaler Vergleich

Keywords: government/public sector; measurement problems; government shares/ratios; international comparison

\section{Einleitung}

Es ist eines der grundlegenden Merkmale der zeitgenössischen Volkswirtschaften, daß darin der Staat (der Öffentliche Sektor, die Öffentliche Hand ...), gemessen an früheren Verhältnissen, eine beispiellose Bedeutung erlangt hat. Dazu können eindrucksvolle Kennziffern für Europa wie für Übersee angeführt werden. (EUROSTAT (b), 1999). Definiert als Anteil an der volkswirtschaftlichen Wertschöpfung ist die Staatsquote in der EU heute nirgends kleiner als 10\% (Vereinigtes Königreich), und erreicht in den skandinavischen Mitgliedsstaaten bis über ein Viertel (Schweden: 26\%); in den USA immerhin auch noch über $18 \%$, nur in Japan bloß um die $8 \%$.

Es bedarf angesichts solcher Größenordnungen keiner ausführlichen Begründung, daß Politik der Frage nach der richtigen Dimensionierung des öffentlichen Sektors und nach seiner Gestion nicht ausweichen kann, und daß dafür soweit irgend möglich objektive Daten erforderlich sind. In diesem Sinne hat die Wirtschaftskammer Österreich (WKÖ) 1999 ein Forschungsprojekt initiiert, und sich damit dieses Problemkreises auf ein neues angenommen, trotz vieler absehbarer sachlicher Schwierigkeiten.

Mit diesem Bericht werden Erkenntnisziel, Ausgangs-Prämissen, theoretische und praktische Fragen der Konzepte und Methoden sowie beispielhafte Ergebnisse des Forschungsprojektes überblicksartig resümiert. Für alle Details muss naturgemäß auf die erarbeiteten Ergebnisse, die maßgebenden Quellen bzw. auch die diskursiven Projektpartien weiterverwiesen werden. Die betreffenden Referenzen werden im Anhang nochmals im Zusammenhang aufgeführt.

\section{Zielsetzung}

Als diese wurde „die Untersuchung der Wirkung unterschiedlicher regulativer und organisatorischer Arrangements bei der Erfüllung öffentlicher Aufgaben auf makroökonomische Indikatoren“ bestimmt (Teufelsbauer, 1999). Als solche Indikatoren sollten jedenfalls untersucht werden: Abgabenquote/Fiskalquote, Staatsausgabenquoten einschl. mandatorischer Elemente, öff. Beschäftigten-Quoten, Verschuldungsquoten; und zwar für Schweiz (CH), Niederlande (NL), Vereinigtes Königrech (UK), Schweden (S) als Vergleichsländer mit Österreich (später miteinbezogen auch Deutschland (D), nach Möglichkeit weitere EU-Mitgliedsländer, sowie USA u. Japan).

In methodischer Hinsicht, die hier von der Zielsetzung nicht zu trennen ist, wurde die Ausarbeitung eines theoretischen Rahmens auf Basis der Volkswirtschaftlichen 
Gesamtrechnung (VGR); eine einschlägige Feldforschung, ggf. auch mit Kontaktnahmen bei den betreffenden Referenzländern; und schließlich die Adjustierung der vorgefundenen Makro-Indikatoren im Hinblick auf Vergleichbarkeit („Benchmarking“) vorgesehen.

Der differenzierten Struktur von Erkenntnisziel und zielbezogener Methodologie entsprach auch die organisatorische Struktur des Projektes, wobei demgemäß zu der Bearbeitung auf der fachlich-theoretischen Ebene umfangreiche statistisch-empirische Bearbeitungen dazutreten sollten. Vor allem in letzterer Hinsicht muß der Beitrag des Institutes für Höhere Studien, Wien (IHS), besonders genannt werden.

\subsection{Begriffliches}

Wie bei solchem Forschungsprojekt zu erwarten, ergab sich sofort eine Reihe von klärungsbedürftigen begrifflichen und definitorischen Vorfragen.

Diese Art von Vorfragen betrifft zunächst die thematisch-inhaltliche Seite: „Öffentlicher Sektor“ - was ist das eigentlich? Hier konnte es natürlich nicht darum gehen, den ganzen Raum der darauf möglichen Antworten auszuloten. Aber auch eine ad hoc - Lösung wäre nicht entsprechend, vor allem wenn man an das Vergleichsziel denkt. Von da her wird einem eine Konzeption à la kleinstes gemeinsames Vielfaches (Maximum) bzw. größter gemeinsamer Nenner (Minimum) nahegelegt erscheinen (Franz, 2000 (c), S. 2); und umso mehr so, wenn auch die statistische Quellenlage mit ins Kalkül gezogen wird. Die letztere Beschränkung ergibt sich nicht nur aus pragmatischen Überlegungen, sondern direkt aus der Projektzielsetzung (s.o.); sie wirkt nach den gegebenen Verhältnissen in hohem Maße fokussierend, nämlich allgemein zugunsten Konzentration auf das Quantifizierbare; und speziell zugunsten der Volkswirtschaftlichen Gesamtrechnung (VGR) samt ihrer Annex-Systeme (insb. die sog. „Satelliten“) (UN etc. 1993). Die VGR liefert insgesamt nicht nur flächendeckendes Zahlenmaterial, sondern auch ein Begriffsgerüst. ${ }^{1}$ Man kommt darum auch umso weniger herum, als dieses Rüstzeug im EU-Rahmen neuerdings mit normativer Kraft ausgestattet worden ist.

Damit soll nicht behauptet werden, daß so alle praktischen Erfordernisse der Vergleichbarkeit schon eingelöst worden sind, noch daß es in diesem Rahmen nicht noch weiterer Konkretisierung bedarf, was den Vergleich selbst (das „,comparandum “) anbelangt. Solche Problematik ist gerade bei der gegenständlichen Studie in erheblichem Umfang aufgetaucht (vgl. unten 4.3).

Zunächst ist nämlich zu entscheiden, ob es sich um einen Kompetenzvergleich handeln soll (= Ausmaß, zu dem sich der Staat involviert, also sich der Anliegen des Bürgers annimmt); oder einen Instrumentalvergleich (= Wege, auf denen er seine Aufgaben wahrnimmt); oder einen Effizienzvergleich (= Wirtschaftlichkeit, die er bei der Aufgabenbesorgung erreicht); oder womöglich um alle 3 Varianten zugleich. Statistisch gesehen ist zumindest der Effizienzvergleich nach wie vor prekär, weil nach der Konvention der VGR der Löwenanteil der Leistungen des „Staates“ (einmal salopp unpräzise hingesagt) ,zu den Kosten bewertet“ wird, und Produktivität, Effizienz von daher so nicht wirklich zu messen sind. ${ }^{2}$

Wenn man jedem dieser drei Gesichtpunkte eine Raumdimension zuordnet, läßt sich die Vergleichssituation als ein Quader veranschaulichen: Diagramm 1 ( Franz, 2000 (a), S. 16). 
Da konkreter Staat sich hinsichtlich der Kompetenzen („Breite“) wie der Instrumentierung („Höhe“) nach wie vor nur rudimentär an bestimmte, international verbindliche Standards halten braucht, wird unmittelbar klar, daß jeder Vergleich unter Einschränkungen leidet: definiert man im Sinne des Minimums (größter gemeinsamer Nenner), bleibt manches außen vor, was in concreto vom Staat besorgt werden mag; im Sinne des Maximums (kleinstes gemeinsames Vielfaches) kommt manches herein, was in concreto von bestimmtem Staat gar nicht besorgt wird. Es kommt so - ähnlich wie beim Index-Vergleich - ein fiktives Element ins Spiel, das den konkreten Vergleichsgegenstand von der Wirklichkeit verschieden macht. Es wird auch noch zu zeigen sein, dass es weiterer fiktiver Elemente bedarf, um Vergleichbarkeit im Sinne des gegenständlichen Erkenntniszieles zu erlangen (sh. weiter unten Pkt. 2.2, zum „Konsolidierungsproblem“").

Mit diesem primären Befund läßt sich immerhin eine erste Systematisierung der methodischen Ansatzpunkte erreichen: Diagramm 2 (Franz, 2000 (c), S. 3) .

Um konkret $\mathrm{zu}$ vergleichen, ist in diesem Rahmen eine m.o.w. fiktive (,ideale“) staatliche Agenda zu definieren, wofür qualitative begriffliche Konkretisierungen aus den betreffenden (internationalen) System-Klassifikationen bzw. den nationalen Budget-Nomenklaturen zu bestimmen und sodann die numerischen Befunde ebenso aus den Budgets bzw. (soweit schon weiterverarbeitet) aus der VGR heranzuziehen sind. Nach Sachlage (Interessenlage) mag die in Vergleich gezogene Agenda auch sehr viel enger sein als das vorgestellte Minimum/Maximum: z.B. nur das Steuerwesen, oder die Sozialpolitik, vorausgesetzt, man erkennt ihnen eine Signifikanz für die Größe des öffentlichen Sektors zu. Es werden solche Quoten in der Folge des näheren angesprochen.

Zur Begrifflichkeit des öffentlichen Sektors gehören auch zwei Konzepte, die v.a. für den nichtmonetären Vergleich von besonderer Bedeutung sind: Öffentliche Aufgaben/Staats-Funktionen und Öffentliche Dienste/Staats-Aktivitäten (jeweils synonym). Staatsfunktionen sind gegenüber Staats-Aktivitäten das allgemeinere oder umfassendere Konzept, dem Begriff ,Staatsziele“ nahekommend. Aktivitäten sind aber dadurch ausgezeichnet, daß es dabei immer auch um Beschäftigte des Staates geht. Diese arbeiten in Dienstellen, die als relativ kleine, betriebsartige „Statistische Einheiten“" (= Elemente der statistischen Erhebung und Auswertung) gelten können, die man daher auch statistisch ,körnig“ beobachten, klassifizieren und auswerten kann - ein wichtiger Umstand für die Sicherung der Vergleichbarkeit, was Aktivität, Input und Output etc. anbelangt. Auf solchen Unterschieden baut die „Ebenen-Theorie“ (Franz, 1994, S. 51ff) auf, wonach angewendet auf den öffentlichen Sektor die öffentlichen Aktivitäten (öffentliche Dienste) gewissermaßen der Unterbau mit den effektiv ablaufenden Arbeitsprozessen sind, worüber die Ebene des „Staates“ als verantwortlich steuernder und finanzierender Überbau darüberliegend zu denken ist, was eo ipso auch für die betreffenden Informationsstrukturen gilt.

Damit ergibt sich auch noch ein Kongruenzproblem hinsichtlich der Deckungsgleichheit der beiden Ebenen insgesamt und hinsichtlich der inneren Strukturen (z.B. Kompetenzkatalog), das bei den Vergleichen ebenfalls zu beachten ist (Franz, 2000 (a), S. 4); (Franz, 2000 (c), S. 21). - Damit einher geht weiters auch der „Trade off“" zwischen höherer Detailschärfe der untersten Ebene gegenüber größerem Informationsumfang der höheren Ebene (Franz, 1994, S. 8). 
Solche Analyse der Situation auf der Seite des realen Vergleichsgegenstandes bringt also noch vor jeder empirischen Erhebung einige ganz wesentliche Umstände ans Tageslicht (Franz, 2000 (c), S. 18 f):

Den Umfang des öffentlichen Sektors („des Staates“) eindeutig oder allgemein gültig bestimmende Kriterien gibt es nicht.

Was damit verbleibt, ist ein formaler Staatsbegriff, manifestiert an der Existenz eines Akteurs, der in gewissem (aber durchaus variablen) Umfang öffentliche Gewalt ausübt.

Auch die VGR (und ihre assoziierten Rechenwerke und internationalen Klassifikationsstandards) können „Staat" letztlich nur in den Grenzen des Zutreffens solcher formaler Kriterien erfassen. ${ }^{3}$

\subsection{Methodisches}

Die Anwendbarkeit des Staatsbegriffes allein ist damit zwar eine notwendige, aber keineswegs eine hinreichende Bedingung der Vergleichbarkeit. Es bedarf für diesen Zweck weiterer Adaptierungsschritte, also gewisser Zu- oder Abrechnungen, wie sie von den unterschiedlichen Kompetenz- und Instrument-Strukturen vorgezeichnet sind.

Eine andere Kategorie von Adaptierungsschritten ist eher buchungs-/verrechnungstechnischer Natur. Zum einen gibt es eine oft ganz beachtliche Masse von verrechnungstechnisch nicht ausdrücklich in Erscheinung tretenden Vorgängen, die aber wirtschaftlich gesehen genau wie Einnahmen bzw. Ausgaben wirken. Der wichtigste Fall sind im Erhebungsverfahren der Steuern schon berücksichtigte Abzugsposten, die das Steueraufkommen vermindern, sei es direkt oder via Bemessungsgrundlage (implizite/indirekte „Förderungen“).

Zum anderen gibt es interne Verrechnungen (Einnahmen-/Ausgaben-Buchungen) zwischen verschiedenen Verwaltungszweigen der jeweiligen Staatsebene und/oder sogar zwischen verschiedenen Rechtsträgern (Ebenen). Derartige Verrechnungen werden für Gesamtdarstellungen normalerweise „konsolidiert“ (weggekürzt) werden. Auch sonst gibt es Netto-Stellungen von Buchungspositionen je der selben Art. Dadurch ist aber das Gesamtniveau der Einnahmen- wie der Ausgabenseite eo ipso beeinflusst.

Solche Vorgänge sind $\mathrm{ab}$ einer gewissen Größenordnung vergleichsrelevant und müssen daher rückgängig gemacht oder zumindest einheitlich gehandhabt werden. Das technische Hilfsmittel hiezu ist im ersten Fall die „Verlängerung“ (Hinzurechnung), indem die zunächst vorgefundene Positionenliste um eben jene nicht ausdrücklich zum Vorschein gekommenen Vorgänge ergänzt wird; im zweiten Fall die einheitliche Vorgangsweise, indem rundum (bei allen Vergleichsländern) entweder alle analogen Posten einheitlich „netto“ oder aber „brutto“ gestellt werden.

Mit anderen Worten: das für den internationalen Vergleich herangezogene Datenset muss so gut als möglich abgestimmt und vereinheitlicht sein hinsichtlich der konkreten Staatsfunktionen (Staatsaufgaben) sowie der einzubeziehenden Akteure (Träger der Durchführung), und schließlich der auf den vollständigen, ursprünglichen (wenn auch nicht manifesten) Stand zurückgeführten Ableitungspositionen (Steuern und Verrechnungen). 
Es bedarf daher normalerweise weitgehender Nacharbeiten (Adjustierungen), sowohl aus sachlichen als auch aus formellen Gründen, und sowohl bei den globalen als auch den mehr spezifischen Kennzahlen, bevor man von Vergleichbarkeit sprechen kann.

Diese Bearbeitungsschritte nehmen naturgemäß in einem derartigen Projekt zunächst großen Raum ein, und sind gewiß auch per se von Interesse. Sie dürfen aber mit der Substanz nicht verwechselt werden, wenn es schließlich um den Vergleich geht. Die konkrete Adaptierungsmaßnahme kann natürlich nur in der Auseinandersetzung mit der jeweiligen Quelle bestimmt werden (s.u. 3. zu Diagramm 4). - Verdichtet auf Kennzahlen-Niveau wird man zweckmäßigerweise von unbereinigten vs. bereinigten (mehr oder weniger voll vergleichbaren) Kennzahlen sprechen.

\section{Gesamtrahmen}

Wie schon oben kurz vorweggenommen kommt aus theoretischen, politischen und praktischen Gründen (umfassender Ansatz $a b$ ovo; lange Anwendungstradition; zunehmende Bedeutung für Zwecke der Bemessung internationaler Zahlungen; anerkannter Charakter einer „Eltern“-Funktion in der internationalen Systemfamilie) dem System der VGR (UN; EU) eine vorrangige Rolle zu, worin der Transaktor „Staat“ an sich seinen festen Platz hat. Die betreffenden internationalen statistischen Veröffentlichungen liegen i.d.R. jährlich vor. Allerdings ist es erforderlich, sie hinsichtlich ihrer Leistungsfähigkeit im konkreten Zusammenhang zu befragen, und sie sind auch nicht die einzige in Betracht kommende Quelle.

\subsection{Volkswirtschaftliche Gesamtrechnungen}

Allerdings hält die Rolle der VGR als Datenquelle mit diesem prinzipiellen Anspruch nicht ganz Schritt. Die hier zu machenden Einschränkungen bezüglich der VGR (und ihren assoziierten Rechenwerken) als Quelle beziehen sich auf ihre Leistungsfähigkeit als statistische Quelle (Franz, 2000 (a), S. 7 f). ${ }^{4}$ Davon zu unterscheiden ist ihre Leistungsfähigkeit als ein begrifflicher, informatischer und vor allem analytischer Ordnungsraster, würde doch in weiterer Folge auch an eine integrierte, synoptische Analyse des vielschichtigen Phänomens „Staat“ als ganzes in diesem Rahmen zu denken sein. (Bis zu diesem Punkt ist die gegenwärtige Bearbeitung allerdings nicht annähernd vorgedrungen.) Von dieser System-Architektur der VGR kann immerhin das insgesamt benötigte Begriffsinventar (statistische Einheiten; Variablen; Rechnungsabgrenzung; Kontentechnik und Modell) hergeleitet werden.

Die hauptsächlichen Konzepte dieser Art sind die Etappen des Wirtschaftskreislaufes (Produktion; Einkommen; Verwendung); die Konten (als ein Symbol für die Zwischenbilanzierung in diesem Wirtschaftskreislauf); die Transaktoren (Akteure im Wirtschaftskreislauf; also auch der Staat); die Transaktionen (Buchungspositionen) und die Salden (z.B. Brutto-Inlandsprodukt). Damit können die Informationen der verschiedenen Quellen in den richtigen Zusammenhang gebracht und für die Analyse aufbereitet werden, sogar wenn sie an den geschilderten Defekten laborieren. Mit diesem Ordnungsraster lassen sich für den Staat sodann jene numerischen Ansatzpunkte identifizieren und gegebenenfalls auch statistisch herausfiltern, die für den Größenvergleich unter allen möglichen Aspekten von Bedeutung sein können. 
Die Kontensequenz der VGR für den Sektor Staat ist beispielhaft (stark reduziert) in Diagramm 3 vorgestellt. In diesem Schema finden alle Ansatzpunkte monetärer Kennzahlen Platz:

\author{
Produktion/Konsum \\ Umverteilung \\ Investitionen \\ Verschuldung \& Vermögen
}

Dabei einbezogen sind auch Verlängerungen und Verkürzungen, wie sie aus verrechnungstechnischen Gründen notwendig werden können; nicht aber jene Ergänzungen/Herauslösungen, wie sie zwischen privatem und öffentlichem Sektor erforderlich werden können (Franz, 2000 (c), Diagramm 9).

Die VGR verdankt ihre Prominenz schließlich nicht zuletzt der Bereitstellung der volkswirtschaftlichen Aggregatgrößen (= Salden auf bestimmten gesamtwirtschaftlichen Konten). Sie werden für standardisierte Kennzahlen aller Art herangezogen (insb. BIP-Quoten ${ }^{5}$ ), haben jeweils aber auch direkt eine Entsprechung in der Kontensequenz für den Staat selbst (z.B. Beitrag des Staates zum BIP):

\begin{tabular}{ll}
\hline Gesamtwirtschaft & Staat \\
\hline - BIP & - öffentliche Produktion, brutto und netto (Beitrag \\
& zum BIP) \\
- VGLV & - öffentlicher Konsum \\
- VE & verfügbares öffentliches Einkommen, mit den \\
& Unterposten: \\
& $\circ$ öffentliche Transferausgaben (laufend; insb. \\
& Sozialtransfers/Umverteilung; Kapital); \\
& $\circ$ öffentliche Transfereinnahmen (insb. \\
& Steuern und Sozialbeiträge) \\
- volksw. Sparen & - öffentliches Sparen \\
- VGLV & - öffentliche Investitionen \\
- volksw. Netto-Position & - öffentliche Verschuldung \\
\hline
\end{tabular}

\title{
3.2 Sonstige Quellen
}

Eine zweite Quelle mit Systemcharakter, aber von dzt. noch geringerem Bekanntheitsgrad (und gleichfalls etwas defektem Stand hinsichtlich der Vergleichbarkeitserfordernisse) ist die Internationale Staatsfinanz-Statistik des Internationale Währungsfonds (IWF). Dieser als ein Segment zu- bzw. untergeordnet ist die Steuereinnahmenstatistik der OECD, welche für die praktisch sehr wichtig gewordenen Steuerquoten(oder allgemeiner: Abgabenquoten-) Vergleiche herangezogen wird (vgl. unten 4.1). Vollständigkeitshalber ist im VGR-Kontext auch noch der Internationale Wirtschafts- 
vergleich (ECP, ICP) zu nennen (UN/ECE; OECD; EU). - Alle diese Quellen sind tatsächlich auch für dieses Projekt benutzt worden.

Von besonderer Bedeutung in diesem Zusammenhang sind auch die Beschäftigtenstatistiken, sei es in der herkömmlichen Form von „head counts“, sei es womöglich differenzierter, indem auch das unterschiedliche Ausmaß von Arbeitseinsatz zum Ausdruck kommt (Vollzeitäquivalente). Die begrifflich-konzeptive Seite ist in dieser Richtung symmetrisch zur VGR zu sehen, und es werden die betreffenden Daten auch im gleichen Kontext systematisch gesammelt und präsentiert. Dasselbe gilt allerdings dann auch für den noch nicht zufriedenstellenden Stand der Harmonisierung. - Eine andere nichtmonetäre Größe von Bedeutung als Basis für Kennzahlen ist die Bevölkerungszahl, gleichfalls durchaus mit Problemen der systematischen Vergleichbarkeit befrachtet (z.B. Alters-, Erwerbstätigenstrukturen).

Vor dem vorgestellten Hintergrund der strukturierten Erkenntnisziele und des Analyserasters lassen sich nun die theoretischen Optionen einer solchen Untersuchung und das Ausmaß, zu welchem hier davon zunächst Gebrauch gemacht wurde, systematisch zusammenstellen: Diagramm 4

Damit wird sofort klar, daß der Schwerpunkt bei den universellen Kennzahlen liegt, und daß durchwegs Adaptierungsschritte gesetzt worden sind. Was die Quellen anbelangt, sind solche nationaler Art systematisch weder in originärer noch aufbereiteter Form einbezogen worden (Felderer et al., 2000, S. 17), es wurden vielmehr nur die internationalen Referenzen herangezogen (also eigentlich Quellen indirekter Art; s.o.).

An dieser Stelle muß aber gleichzeitig festgehalten werden, daß auch auf internationaler Ebene eine systematische Auseinandersetzung mit der AdaptierungsProblematik nur in Ansätzen zu finden ist (s.u. 4.1). Angesichts „,nominal“ nicht erkennbarer Defekte kann damit leicht der Eindruck entstehen, daß Vergleichbarkeit herrscht, und also die Daten ohne weiteres zum Nennwert zugrundegelegt werden könnten (z.B. bei Betrachtung der ,originären“ Ergebnisse der VGR zum Staat allein).

Diese beiden Gesichtspunkte - unerläßliche Adaptierungsnotwendigkeiten der vorfindlichen Daten und nach wie vor unglaublich dürftige Beschäftigung mit dieser Problematik auf der internationalen Ebene - werden als wichtige Nebenergebnisse dieses Projektes zu resümieren sein.

\section{Statistischer Befund}

In diesem Abschnitt werden die in diesem Projekt beschrittenen konkreten Zugangswege kurz beschrieben und exemplarische Hauptergebnisse vorgestellt.

\section{1 Abgabenquoten}

Das Gebiet der öffentlichen Abgaben (Steuern, Sozialversicherungsbeiträge, Gebühren) ist international gut dokumentiert (OECD [jährl.]; IWF [jährl.]). Wegen der längerfristig notwendigen Deckung der Staatsausgaben vor allem über öffentliche Abgaben ist es legitim, diese Quoten unter dem universellen Typ zu subsumieren (Felderer et al., 2000, S. 2). 
Wegen der Möglichkeit von Gegen-Verrechnungen (d.h. im Zuge des Steuererhebungsverfahrens) von staatlichen Vergünstigungen, die anders auch im Wege von (expliziten) Transferzahlungen gewährt werden könnten (implizite Zuwendung), und wegen der Option, Sozialversicherungsbeiträge durch privatrechtliche Verträge mit Dritten abzulösen (mandatorische Beiträge), sind allerdings Adaptierungen vonnöten, die in den vorgenannten Quellen zunächst nur in geringem Umfang enthalten sind:

\begin{tabular}{l} 
+ mandatorische Beiträge an den privaten Sektor \\
+ implizite Förderungen \\
\hline = Hinzurechnungen \\
- Steuern von Sozialtransfers \\
\hline \pm Adaptierungssaldo \\
\pm Saldo am Generationenkonto \\
\hline$=$ Gesamtbereinigung \\
\hline
\end{tabular}

In manchen Ländern werden Steuern auch von den Sozialtransfers erhoben (i.S. einer universellen, alle Einkünfte und Zuflüsse berücksichtigenden Bemessungsgrundlage). Man kann in diesem Sinne die ursprüngliche, nominelle staatliche SozialTransferleistung als von Haus aus vermindert ansehen und eo ipso auch das Steueraufkommen. Die Argumentation ist für bestimmte globale Analysen zutreffend, aber bei differenzierterer Sichtweise problematisch (z.B. Finanzausgleichssystem kommt der Steuereingang nicht unbedingt dem Träger der Sozialtransfer-Last zugute; wenn die Steuern später (im Veranlagungswege) eingehoben werden, kann es auch zu Belastungsverschiebungen auf der Empfängerseite kommen. Man mag es als noch problematischer ansehen, wenn auch für indirekte Steuern (KonsumausgabenKomponente) ein Abzug vorgenommen wird.

Die Staaten gehen laufend Pensions-Verpflichtungen (sei es aus allgemeinen politischen Erwägungen, sei es für ihre eigenen Bediensteten) ein, die nicht unbedingt durch die Pensionsbeiträge gedeckt sind. Unter der Annahme der Weitergeltung des Pensionsrechtes von heute sowie demographischer Parameter lassen sich die Über/Unterdeckungs-Beträge ermitteln, die im Referenzjahr entstehen (Saldo am Generationenkonto). Die Einbeziehung solcher Komponenten ist nicht nur aus Gründen der explorativen Neugier gerechtfertigt: sie zeigt besser als manche theoretische Diskussion, daß den Adaptierungen immer etwas Arbiträres, Fiktives anhaftet, wofür man daher nicht ohne weiters Allgemeingültigkeit beanspruchen kann.

In der Übersicht 1 kommt für eine Anzahl von Ländern die Signifikanz der Bereinigungen klar zum Vorschein, wobei der (einebnende) Netto-Effekt besonders ausgeprägt ist. Quelle solcher Berechnung ist die laufende Einkommensstatistik der OECD (OECD ([jährlich]) sowie weitere verdienstvolle Studien in deren Bannmeile (vgl. Felderer et al., 2000, S. 9/Fußnote ${ }^{4}$ ) und S. 17). Daraus erklärt sich auch der eingeschränkte Länderumfang einer solchen übergreifenden Zusammenschau, weil der Kreis der je Komponente einbeziehbaren Länder jeweils mehr oder weniger verschieden ist. Nicht nur vollständigkeitshalber ist auch anzumerken, daß gerade auf dem Gebiete der steuerrechtlichen Ausgestaltung (Steuereinheit; Definition der Bemessungsgrundlage; Wahlmöglichkeiten usw.) eine Vielfalt von Variationen ins Spiel kommt, die in einer solchen Untersuchung umso schwerer auszuschöpfen ist (Felderer et al., 2000, S. 6f). 


\subsection{Transferquoten}

Von dieser ausgabenseitigen Kategorie wurden die Sozialquoten näher untersucht, wobei auch hier Vorarbeiten der OECD von größtem Nutzen waren (SOCX Datenbasis; Adema, 1996, und Adema et al., 1999) ${ }^{6}$.

Die oben unter Pkt. 4.1 vorgeführten Adaptierungen (Leistungen aus mandatorischen Beiträgen, implizite Förderungen; Steuern von Sozialtransfers) kommen hier natürlich analog zum Tragen, womit sich abermals die enge Interaktion von Steuer- und Sozialtransfer-System herausstellt. In dem untersuchten Sample von mehr als der Hälfte der OECD-Länder ist damit allein die Variation der Sozialquote um über die Hälfte reduziert. Anzumerken ist, daß nicht nur die Barauszahlungen, sondern auch Realtransfers berücksichtigt werden (Gesundheitswesen). Mit dieser Abgrenzung folgte man Adema et al., 1999, sie weist aber auf das Problem einer mehr oder weniger willkürlichen Grenzziehung des Sozial-Begriffes hin. ${ }^{7}$

\subsection{Funktionenvergleich}

Solcher kann auf einzelne oder alle Funktionen des Staates abstellen. Im letzteren Fall handelt es sich gleichzeitig um eine Definition des „Staates“, und zwar auf der Ebene der staatlichen Aktivitäten, die sich als Leistungs-Erbringung geltend machen, also im Sinne von „Arbeit“ (nicht also seine Transferleistungen, oder seine Investitionen). Als Gegenstände der Messung kommen demgemäß in Betracht (s.o. 3, zu Diagramm 4):

- Beitrag zum BIP (Wertschöpfung)

- Beschäftigte

Für beide ist im ggstdl. Projekt ein Vergleichbarkeitsversuch unternommen worden. Man ging dabei von der Prämisse aus, daß das staatliche Funktionenbündel von Land zu Land a priori nicht vergleichbar ist, weil Auslagerungen und/oder Zuständigkeitszuweisungen v.a. in Bereichen wie Gesundheit, Bildung und soziale Fürsorge von Land zu Land und auch über die Zeit ganz verschieden ausfallen. Diese sind also im Sinne des Minimal- oder des Maximal- (bzw. eines standardisierten Optimal-) Konzeptes rückgängig zu machen. Die Frage, ob die Adaptierung im Wege einer Hinzurechnung der im einzelnen Landesfall nicht beim Staat enthaltenen Funktion oder umgekehrt im Wege der Abrechnung von nur in diesem Land enthaltenen Funktionen geschehen soll, ist dabei von Haus aus offen, und eigentlich nur arbiträr zu beantworten. Es sollte jedenfalls die BIP- oder Beschäftigungsquoten für diese umfänglich bereinigten Staats-Indikatoren gleichmäßiger ausfallen.

Im Prinzip kann hier sowohl ein Querschnitt- als auch ein Längsschnitt-Vergleich aufschlußreich sein, letzterer vor allem insofern, als institutionelle Veränderungen, welche die aktuelle Verteilung der Staatsfunktionen berühren, durch die Adaptierung neutralisiert werden. Als Beispiel wird hier ein Querschnitt-Vergleich beigegeben (Übersicht 2). ${ }^{8}$ In dieser Hinsicht ist leider eine vorerst noch gar nicht befriedigende statistische Situation festzustellen, und zwar wegen völlig unzulänglicher Gliederungsdetails. In dem Querschnittsvergleich müßten nämlich erwartungsgemäß die breiter 
basierten Quoten stärker konvergieren als die engeren. Diese Erwartungen sind allerdings auf der Ebene der verfügbaren Daten nur zu einem geringen Grade aufgegangen. Solches Ergebnis, wie es sich analog auch im Längsschnitt-Vergleich erwiesen hat, wäre daher zuallererst als ein Anspruch an mehr spezifische Detail-Evidenz zu interpretieren, um Überlagerungen der erwarteten Tendenz zuvorzukommen.

Nicht nur der Vollständigkeit halber soll auch der im Vergleich zur VGR weniger bekannte „Internationale Wirtschaftsvergleich“ angeführt werden (EUROSTAT (a) [jährlich]). Dessen Sinn ist es gerade, Einschränkungen der Vergleichbarkeit durch Umstellung auf Kaufkraftparitäten (was hier nicht von Belang ist) und Vereinheitlichung von Abgrenzungskonzepten der Endverwendung zu beheben. Diese Vergleichsbasis wurde (ähnlich wie zuvor beschrieben) adaptiv erweitert, womit die Differenzen der Rechenkonzepte fortfallen. Was verbleibt, ist ein vergleichsweise viel ausgeprägterer Konvergenzeffekt (Übersicht 3; Franz, 2000 (b)).

Was die Beschäftigungsquoten anbelangt, sind neuere Bemühungen zu vermerken, wonach OECD-weit vergleichbare Zahlen dazu eruiert werden sollen. Vorliegendes Zahlenmaterial auch anderer Provenienz wurde gesichtet, eine wesentlich bessere Vergleichsbasis als gemäß Übersicht 2 (s. o.) kam nur hinsichtlich der Berücksichtigung des privaten Non-profit-Sektors zum Vorschein (Felderer et al., 2000, IV).

\subsection{Bewertung}

Bereinigungen der vorgefundenen Ausgangswerte und darauf basierter Quoten erweisen sich konzeptgemäß als notwendig, und statistisch im Prinzip auch als machbar. Allerdings sind Belege für erwartete statistische Konvergenzwirkungen auf der Ebene des Staates als ganzes noch nicht flächendeckend beibringbar, und eigentlich muß die statistische Flanke des Funktionenvergleiches als noch offen eingestuft werden. Auch sonst sind die involvierten Bearbeitungserfordernisse beträchtlich und ohne eine gezielte, über die routinemäßige Datenbereitstellung im Rahmen der internationalen VGR-Melde-Verpflichtungen hinausgehende Initiative gar nicht zu leisten.

\section{Resümee}

Wie mit dem hier berichteten Projekt lange nicht alle Facetten der angesprochenen Problematik aufgegriffen oder womöglich sogar beantwortet werden konnten, so läßt sich auch mit diesem kurzen Aufsatz nur ein schlaglichtartiger Eindruck von den Prämissen, Konzepten und Ergebnissen vermitteln. Wenn dazu noch ein Resümee versucht werden soll, wird man schnell bei Überlegungen vom Typ „de lege ferenda“ landen.

Schon in der ersten Phase der Studie ist klar geworden, daß es sich dabei keineswegs um eine triviale Aufgabe handelt, weder was das Gewicht solcher Aussagen anbelangt, noch was die dabei auftauchenden definitorischen und konzeptiven Schwierigkeiten betrifft. Insbesondere ist die Mehrdimensionalität des öffentlichen Sektors zu berücksichtigen.

Die Quellenlage ist ein Problem sui generis, und vorderhand noch der eigentliche Engpass. VGR ist und bleibt eine zentrale begriffliche Referenz; und sie wäre eine 
wichtige, wenn nicht die wichtigste statistische Referenz, kann aber in letzterer Hinsicht mit den aktuellen Zahlenbildern noch keineswegs zufriedenstellen.

Dementsprechend werden den angezielten „Vergleichsaussagen“ zwangsläufig weiterhin relativ umfangreiche Vorarbeiten zur Erarbeitung einer entsprechend aufbereiteten oder „bereinigten“ Datenbasis vorgelagert sein. Diese Vorarbeiten dürfen nicht mit dem Hauptzweck dieses Projektes verwechselt werden, sie sind aber eine der unabdingbaren Voraussetzungen des Vergleiches.

Unter diesen Vorraussetzungen lassen sich immerhin schon beim status quo der Bearbeitung interessante exemplarische Aussagen treffen.

- Der Bereinigungseffekt ist statistisch signifikant (d.h. ein Vergleich von Staatsquoten ohne solche Bereinigung wäre sicher nicht haltbar).

- Die maßgeblichen zur Bereinigung heranstehenden Unterschiede ergeben sich einerseits aus unterschiedlichen Auslagerungspolitiken (wer macht was?), andererseits aus unterschiedlichen Verrechungstechniken (vor allem im sozialpolitisch relevanten Bereich).

- Obwohl verschiedene Quoten-Varianten errechenbar und auch entsprechend bereinigbar sind, ist der Datenbefund unbefriedigend bzw. werden Initiativen zur Weiterentwicklung nahegelegt.

- Die bereinigten Quoten zeigen im Vergleich mit den unbereinigten eine Tendenz zur Konvergenz (d.h. die Unterschiede in der Größe des öffentlichen Sektors sind nicht so ausgeprägt wie von den rohen Staatsquoten suggeriert).

- Dem Phänomen „öffentlicher Sektor“ ist mit einer einzigen Maßzahl kaum beizukommen, es bedarf einer Mehrzahl von die verschiedenen Dimensionen abtastenden Kenngrößen.

Das Resümee vom Resümee: es liegt angesichts des aktuellen, oft unqualifizierten Umgangs mit Zahlen zur Größe des Staates ein legitimes Anliegen wissenschaftlicher Untersuchung und Klarstellung vor. Von nationaler Warte aus ist dem Problem der hier so wichtigen Vergleichbarkeit nicht beizukommen. Eine breitere Initiative - sei es als spontane Zusammenarbeit einiger Pionier-Länder, sei es auf inter- oder supranationaler Ebene - wäre daher angesagt. Oder anders herum: Niemand kann beim statistischen „state of the arts“ ernsthaft behaupten, daß er unangreifbare empirische Belege für normative Aussagen über „exzessive“ Größe des Öffentlichen Sektors und/oder über „Produktivitätsdefizite“ und dergleichen hätte.

\section{Anmerkungen}

Vgl. ESA-Rats-Verordnung (EG) Nr. 2223/96 v. 25. Juni 1996.

${ }^{2}$ Hier gibt es neuere Initiativen zu einer Abhilfe unter den Auspizien der OECD (sog. PUMA-Projekt), mit dazugeschalteten nationalen Affiliationen; und auch seitens der EU im Rahmen der EUROSTAT-Arbeitsgruppe für VGR. Die Ergebnisse sind abzuwarten. 
${ }^{3}$ Ein solches ist neuerdings das Kriterium der 50\%-Betriebskostendeckung (ESA 1995, para. 3.27ff: EUROSTAT 1995).

${ }^{4}$ Dort sind auch weitere beim status quo der Ergebnisse zu verzeichnende Mängel angeführt.

5 Vor Abzug von Produktsteuern (netto) und imputierter Bankgebühr: „Wertschöpfungsquote“ (aus Konsistenzgründen zu bevorzugen); vgl. Franz, 2000 (b), S. 7ff.

${ }^{6} \mathrm{Da}$ die Problematik im Sozialbereich fokussiert, ist auch beim Alpbach-Seminar, 22 0800 , unterstrichen worden.

${ }^{7}$ Vgl. das sog. ESSOSS-Projekt der Europäische Kommission (EUROSTAT (d)).

${ }^{8}$ Die ,sonstigen“ marktbestimmten Dienstleistungen sind definiert als B 29/74; die sonstigen nicht marktbestimmten (ONMS) als B 36/- (s. EUROSTAT (b)). 


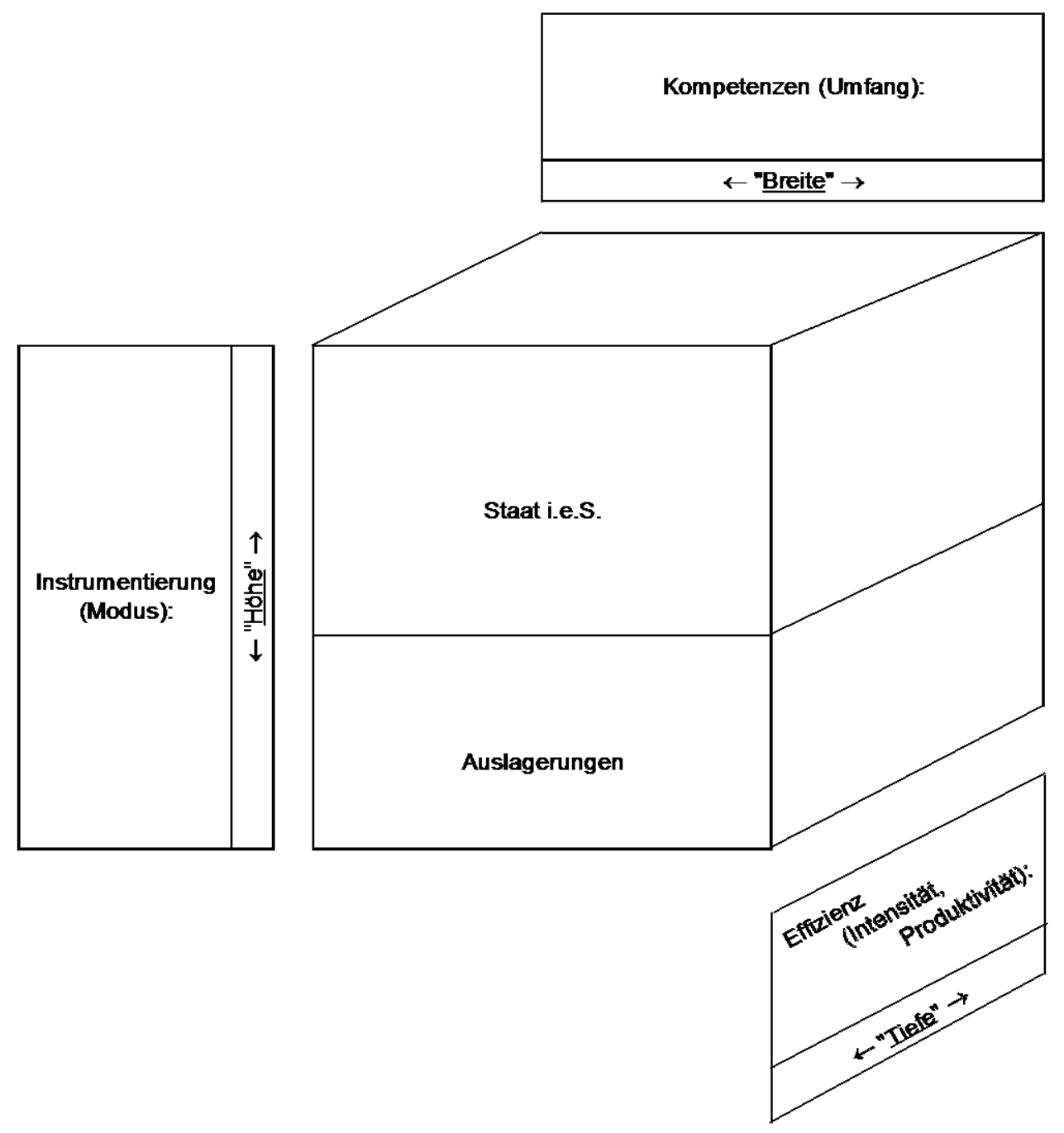

Abbildung 1: Dimensionen des statistischen Staatsvergleiches 


\begin{tabular}{|c|c|c|c|c|}
\hline & \multirow{2}{*}{$\begin{array}{l}\text { Vergleichsgegenstand } \\
\text { (Vergleichbarkeits- } \\
\text { Erfordernis) }\end{array}$} & \multicolumn{2}{|c|}{ Vergleichs-Methode } \\
\hline & & & $\begin{array}{l}\text { Minimum- } \\
\text { Maximum }\end{array}$ & Kennzahl \\
\hline \multirow{3}{*}{ 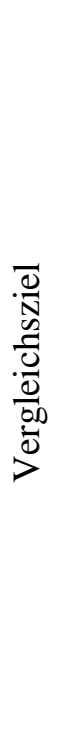 } & $\begin{array}{l}\text { Kompetenz } \\
\text { (Umfang) }\end{array}$ & $\begin{array}{l}\text { Aufgaben (= Ausgaben): } \\
\text { Umfangsunterschiede } \\
\text { vergleichen! }\end{array}$ & fiktiv ("ideal") & $\begin{array}{l}\text { Anteile (BIP, } \\
\text { u.s.w.) }\end{array}$ \\
\hline & $\begin{array}{l}\text { Instrumentalität } \\
\text { (Umsetzungswege) }\end{array}$ & $\begin{array}{l}\text { Aktivitäts-(=Aufgaben-) } \\
\text { Komponenten: } \\
\text { Instrumenten-Mix } \\
\text { untersuchen! }\end{array}$ & fiktiv-real & Strukturen \\
\hline & $\begin{array}{l}\text { Effizienz } \\
\text { (Wirtschaftlichkeit) }\end{array}$ & $\begin{array}{l}\text { Aktivität- } \\
\text { (=Ressourceneinsatz): } \\
\text { Umfangs-Unterschiede } \\
\text { ausschalten! }\end{array}$ & real & $\begin{array}{l}\text { Produktivitäten, } \\
\text { Intensitäten u.ä. }\end{array}$ \\
\hline
\end{tabular}

Abbildung 2: Ziele- x Methoden-Systematik des Staatsvergleiches 


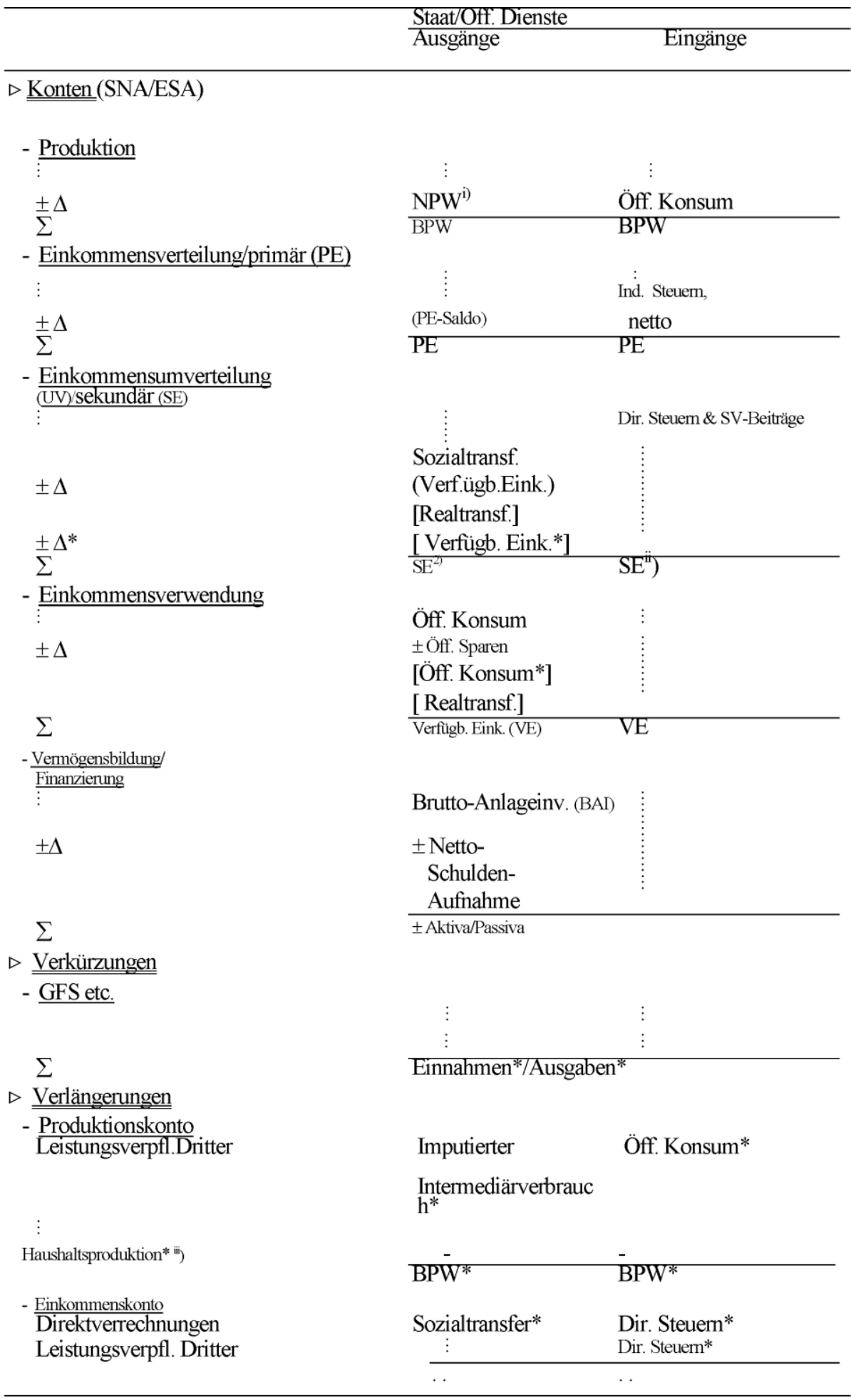

Legende: ${ }^{\text {i) }}$ untergliederbar: BEUA, Afa...

ii) $=$ Umverteilungsmasse

iii) soweit von Haushalten Leistungen erbracht werden, die anderswo (ansonsten) Staat/öffentl. Dienste besorgen

$*$ adjustiert $\quad \Delta$ Saldo-Größe $( \pm)$

Abbildung 3: Kontensequenz der VGR 


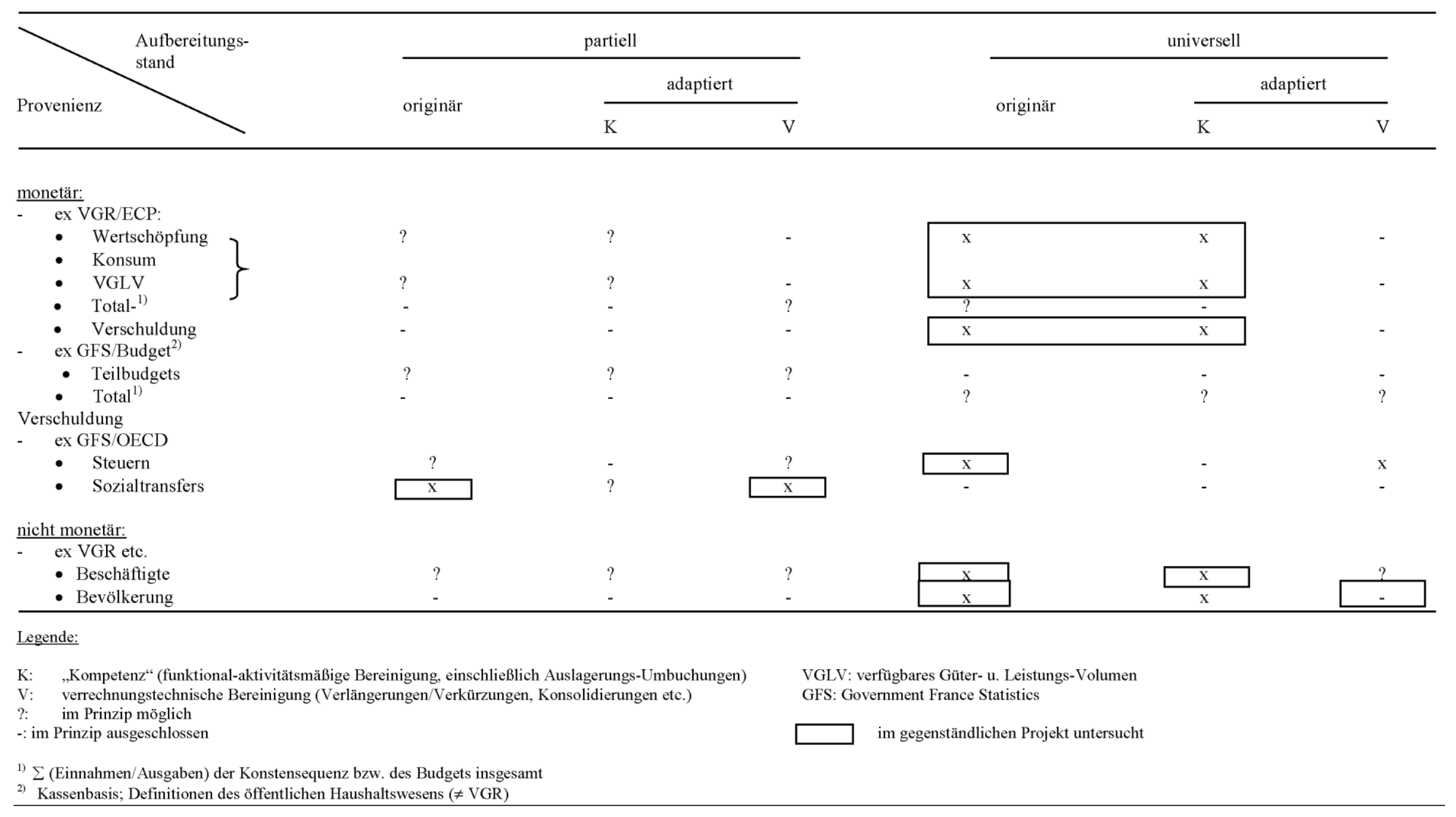

Abbildung 4: Optionen der Untersuchung mit ihren Datenquellen 
Übersicht 1: Bereinigte Abgabenquoten (1995; in Prozent vom BIP)

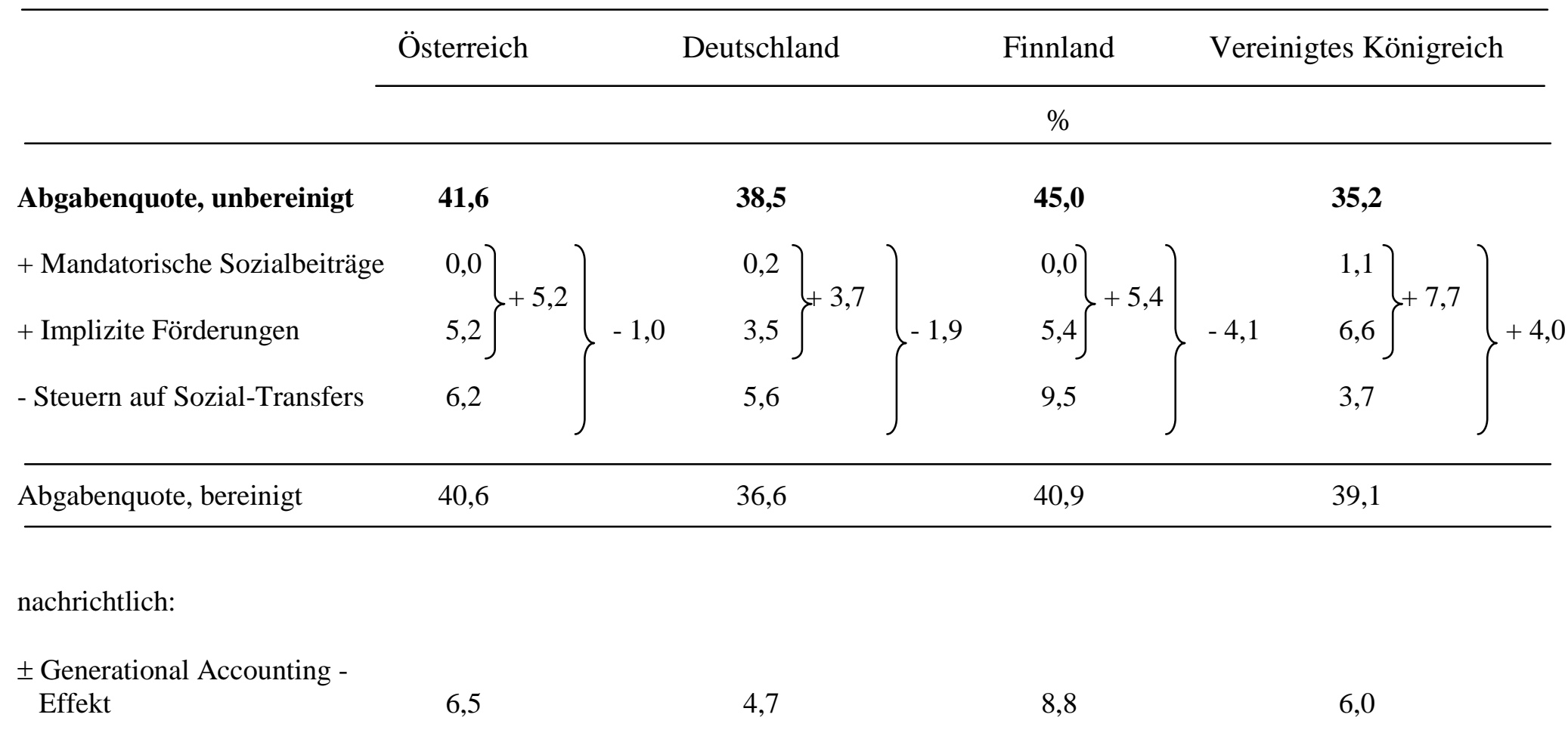


Übersicht 2: Funktionale Kennzahlen "Staat" - Querschnitt - Vergleich

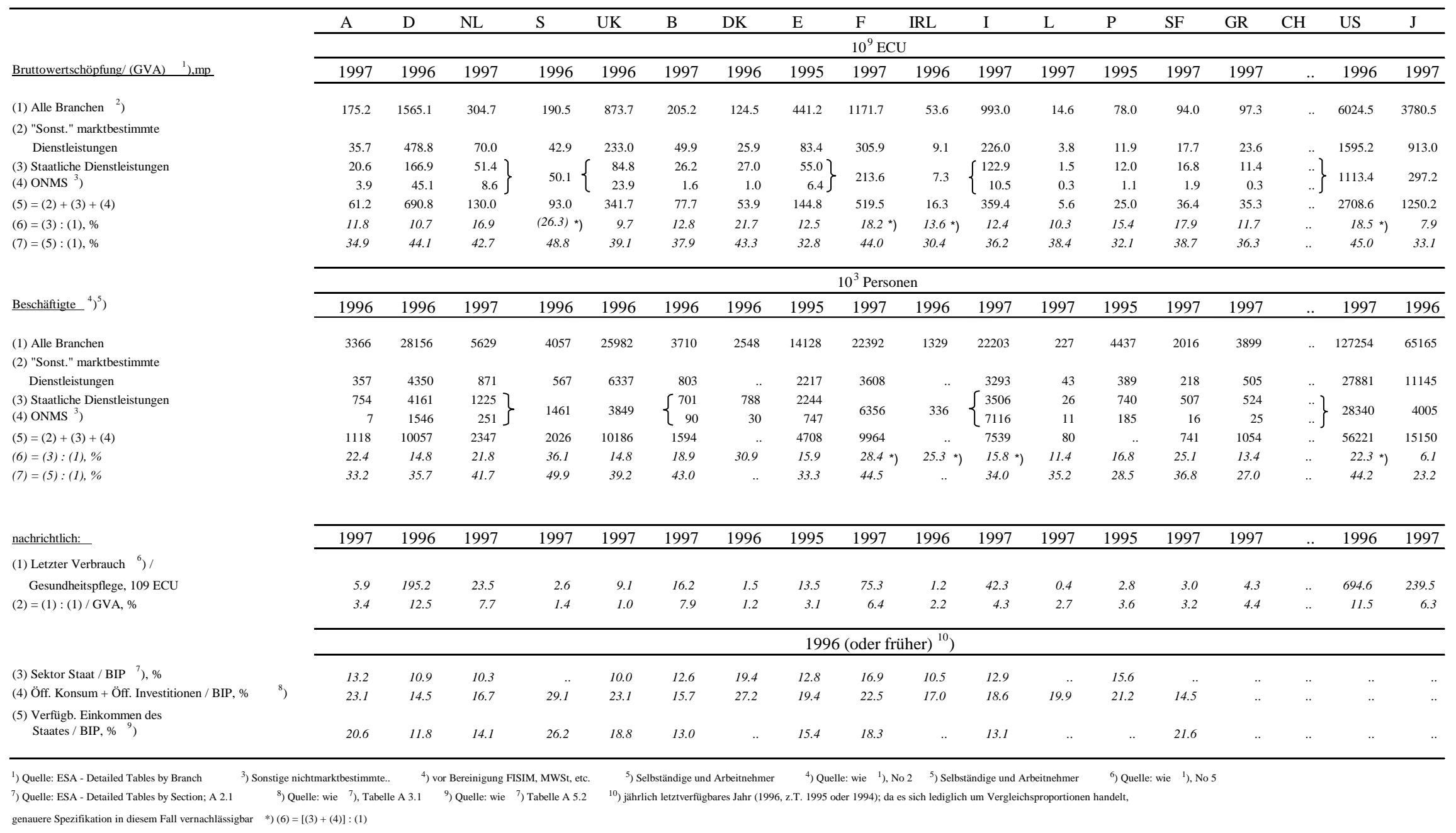


Übersicht 3: Funktionale Kennzahlen "Staat" - Querschnitt - Vergleich (Analytischer Zwischenbericht);

Europäischer Wirtschaftsvergleich (ECP) 1997 - Ausgangsdaten ("Tabelle 1")

\begin{tabular}{|c|c|c|c|c|c|c|c|c|c|c|c|c|c|c|c|c|}
\hline & A & D & NL & $\mathbf{S}$ & UK & B & DK & E & $\mathbf{F}$ & IRL & I & $\mathbf{L}$ & $\mathbf{P}$ & SF & GR & CH \\
\hline & \multicolumn{16}{|c|}{ Reale Pro-Kopf-Ausgaben / KKP, in $€$} \\
\hline $\begin{array}{l}\text { (1) Ausgaben d. Staates } \\
\text { f. d. Letztverbrauch }\end{array}$ & 4118 & 2089 & 2979 & 5519 & 3713 & 3001 & 6033 & 2528 & 3923 & 2675 & 2859 & 2878 & 3901 & 4023 & 2075 & 2726 \\
\hline \multicolumn{17}{|l|}{ (2) Ex priv. Konsum } \\
\hline o Gesundheit & 476 & 2285 & 2106 & 207 & 212 & 1736 & 236 & 384 & 1405 & 376 & 707 & 1109 & 335 & 458 & 613 & 1468 \\
\hline o Unterhaltung, Kultur & 512 & 378 & 428 & 385 & 452 & 191 & 448 & 166 & 274 & 350 & 255 & 262 & 266 & 359 & 210 & 347 \\
\hline o Unterricht & 55 & 140 & 83 & 13 & 192 & - & 230 & 253 & 66 & 448 & 77 & 6 & 160 & 46 & 158 & 164 \\
\hline o p IOE & 370 & 368 & - & 272 & 452 & - & 212 & 108 & 53 & - & 49 & 599 & 185 & 354 & 40 & 700 \\
\hline$(3)=(1)+(2)$ & 5531 & 5260 & 5596 & 6396 & 5021 & 4928 & 7159 & 3439 & 5721 & 3849 & 3947 & 4854 & 4847 & 5240 & 3096 & 5405 \\
\hline
\end{tabular}

\section{B. ICP "Originär"}

\begin{tabular}{|c|c|c|c|c|c|c|c|c|c|c|c|c|c|c|c|c|}
\hline $\begin{array}{l}\text { (1) Ausgaben d. Staates } \\
\text { f. d. Letztverbrauch }\end{array}$ & 1886 & 1022 & 1644 & 1859 & 1579 & 1742 & 984 & 1612 & 1667 & 1020 & 1413 & 1383 & 1925 & 1350 & 1288 & 1447 \\
\hline \multicolumn{17}{|l|}{ (2) Ex Individualverbrauch } \\
\hline o Gesundheit & 1294 & 2347 & 2121 & 1197 & 1180 & 1718 & 1354 & 765 & 2095 & 1148 & 1240 & 1231 & 736 & 1199 & 965 & 1696 \\
\hline o Unterhaltung, Kultur & 526 & 464 & 495 & 668 & 546 & 196 & 1296 & 172 & 411 & 514 & 305 & 294 & 305 & 525 & 230 & 414 \\
\hline o Unterricht & 1289 & 779 & 995 & 1041 & 896 & 1238 & 1705 & 776 & 1156 & 1046 & 796 & 1120 & 1569 & 1092 & 486 & 956 \\
\hline o p IOE & - & - & - & - & - & - & - & - & - & - & - & - & - & - & - & - \\
\hline
\end{tabular}

Quelle: Kaufkraftparitäten und verwandte Wirtschaftsindikatoren, Ergebnis für 1997, EUROSTAT, 
Übersicht 3: Größe des Öff. Sektors - Funktionen-Vergleich (Analytischer Zwischenbericht);

Europäischer Wirtschaftsvergleich (ECP) 1997 - Rekonziliation ("Tabelle 2")

\begin{tabular}{|c|c|c|c|c|c|c|c|c|c|c|c|c|c|c|c|}
\hline & $\mathbf{A}$ & D & NL & $\mathbf{S}$ & UK & B & DK & E & $\mathbf{F}$ & IRL & I & $\mathbf{L}$ & $\mathbf{P}$ & $\mathbf{S F}$ & GR \\
\hline & \multicolumn{15}{|c|}{ Reale Pro-Kopf-Ausgaben / KKP, in $€$} \\
\hline$\sum((3)=(1)+(2))$ & 5531 & 5260 & 5596 & 6396 & 5021 & 4928 & 7159 & 3439 & 5721 & 3849 & 3947 & 4854 & 4847 & 5240 & 3096 \\
\hline \multicolumn{16}{|l|}{ B. ICP "originär" (= publizierte Version) } \\
\hline$\sum((3)=(1)+(2))$ & 4995 & 4612 & 5255 & 4765 & 4201 & 4894 & 5339 & 3325 & 5329 & 3728 & 3754 & 4028 & 4535 & 4166 & 2969 \\
\hline \multicolumn{16}{|l|}{ C. ICP ("Tatsächlich") } \\
\hline (1) $\sum \mathrm{B}$ & 4995 & 4612 & 5255 & 4765 & 4201 & 4894 & 5339 & 3325 & 5329 & 3728 & 3754 & 4028 & 4535 & 4166 & 2969 \\
\hline (2) - Soziales & 82 & 296 & 282 & 1189 & 312 & - & 1539 & - & 288 & 70 & 106 & 181 & 108 & 615 & 112 \\
\hline$-p \operatorname{IOE}$ & 370 & 368 & & 272 & 452 & - & 212 & 108 & 53 & - & 49 & 599 & 185 & 354 & 40 \\
\hline$\sum((3)=(1)+(2))$ & 5447 & 5276 & 5537 & 6226 & 4965 & 4894 & 7090 & 3433 & 5670 & 3798 & 3909 & 4808 & 4828 & 5135 & 3121 \\
\hline \multicolumn{16}{|l|}{ D. Differenzen } \\
\hline$\overline{\left.\sum \mathrm{C}-\sum \mathrm{B}^{1}\right)}$ & 536 & 648 & 341 & 1631 & 820 & 34 & 1820 & 114 & 392 & 121 & 193 & 826 & 312 & 1074 & 127 \\
\hline$\Sigma\left(-\sum A^{2}\right)$ & 84 & -16 & 59 & 170 & 56 & 34 & 69 & 6 & 51 & 51 & 38 & 46 & 19 & 105 & -25 \\
\hline E. Kennzahlen: Anteil "Staat" an der & \multirow{2}{*}{\multicolumn{15}{|c|}{$\%$}} \\
\hline Wertschöpfung & & & & & & & & & & & & & & & \\
\hline Tabelle I A. (1) & 19.0 & 11.0 & 15.3 & 25.3 & 20.1 & 14.9 & 24.9 & 20.8 & 19.6 & 15.2 & 16.8 & 8.3 & (45.7) & 22.0 & 27.2 \\
\hline (3) & 25.5 & 27.6 & 28.7 & 29.3 & 27.2 & 24.5 & 29.4 & 28.3 & 28.6 & 21.9 & 23.3 & 14.0 & (56.8) & 28.7 & 33.4 \\
\hline$-"-1$ B. (1) & 8.7 & 5.3 & 8.4 & 8.5 & 8.5 & 8.6 & 4.0 & 13.3 & 8.3 & 5.8 & 8.3 & 4.0 & (22.5) & 7.4 & 13.9 \\
\hline (3) & 23.0 & 24.2 & 26.9 & 21.8 & 22.7 & 24.3 & 21.9 & 27.3 & 26.7 & 21.2 & 22.1 & 11.6 & (53.1) & 22.8 & 32.0 \\
\hline$-"-2$ C. (3) & 25.1 & 27.7 & 28.4 & 28.5 & 26.9 & 24.3 & 29.1 & 28.2 & 28.4 & 21.6 & 23.1 & 13.9 & (56.6) & 28.1 & 33.6 \\
\hline
\end{tabular}

Quelle: s. "Tabelle 1"

1) Gründe der Differenzen:

(1) Fälle von "Basic Headings" (= unterste Gewichts-Posten; p IOE u. Soziales), die in ECP in "Sonstige Güter \& Dienstleistungen" enthalten sind und daher nirgends sonst zum Vorschein kommen.

(2) Nicht- Additivität der EKS-Ergebnisse (Reale Werte)

2) Gründe der Differenzen: Nicht-Additivität der EKS-Ergebnisse

${ }^{3}$ ) Angaben zur Wertschöpfung nicht verfügbar. 


\section{Literatur}

(In den Referenzen Felderer et al., Aug. 2000, und Franz (a) - (c) zitierte Literatur ist hier nicht eigens angeführt.)

W. Adema. Net Social Expenditure, Labor Market and Social Policy Occasional Papers, No. 39, OECD, Paris, 1999.

W. Adema, M. Einerhand, B. Eklind, J. Lotz, and M. Pearson. Net Social Expenditure, Labor Market and Social Policy Occasional Papers, No. 19, OECD Paris, 1996.

EUROSTAT. European System of Accounts, ESA, 1995, Luxembourg, 1999.

EUROSTAT (a). Kaufkraftparitäten und verwandte Wirtschaftsindikatoren, Ergebnisse für 19..., jährlich.

EUROSTAT (b). Volkswirtschaftliche Gesamtrechnungen-ESVG, Detaillierte Tabellen nach Produktionsbereichen, Luxembourg, jährlich.

EUROSTAT (c). Volkswirtschaftliche Gesamtrechnungen-ESVG, Detaillierte Tabellen nach Sektoren, Luxembourg, jährlich.

EUROSTAT (d). Social protection expenditure and receipts: European Union, Iceland and Norway, Data 1980-96, EUROSTAT, Luxembourg 1999 (abbr. ESSOSS).

EUROSTAT (e). Volkswirtschaftliche Gesamtrechnungen-ESVG, Detaillierte Tabellen nach Produktionsbereichen, Luxembourg, jährlich.

B. Felderer, B. Grossmann und R. Komann. Die Größe des Öffentlichen Sektors. Projektbericht, IHS, Wien/Alpbach, August 2000.

A. Franz (a). Die Größe des Öffentlichen Sektors: Determinanten und Vergleiche. Ein Forschungsthema - Ein Forschungsansatz, Wien, Juli 2000, Paper für das WKÖBenchmarking-Projekt, Alpbach 22/23, August 2000.

A. Franz (b). Die Größe des Öffentlichen Sektors: Funktionen-Vergleich, WirtschaftsVergleich: Analytischer Zwischenbericht, Wien, Juli 2000.

A. Franz (c). Die Größe des Öffentlichen Sektors, Konzept-Papier für das WKÖBenchmarking-Projekt, Eröffnungsdiskussion, Wien, Jänner 2000.

A. Franz. Volkswirtschaftliche Gesamtrechnungen. Das statistische System der Makroökonomie, Österr. Studien zur Amtlichen Statistik, No. 1, ÖSTAT, Wien,1994.

Government Finance Statistics, IMF, Washington, jährlich.

Revenue Statistics, OECD, Paris, jährlich.

System of National Accounts 1993, EC, IMF, OECD, UN, WB, New York etc., 1993 (kurz: SNA 1993).

W. Teufelsbauer. Projektskizze v. Nov. 99 (intern). 
Adresse des Autors:

Alfred Franz

Statistik Österreich

Hintere Zollamtsstrasse $2 \mathrm{~b}$

A - 1030 Vienna

Phone: (+ 43 1) 711287300

Fax.: (+43 1) 7185060

Email: alfred.franz@statistik.gv.at 